\title{
PRESSURE DISTRIBUTION OF A HORIZONTAL WELL IN A BOUNDED RESERVOIR WITH CONSTANT PRESSURE TOP AND BOTTOM
}

\author{
J. J. Orene ${ }^{1, *}$ and E. S. Adewole ${ }^{2}$ \\ 1, 2, Department of Petroleum Engineering, University of Benin, Benin City, Edo State, Nigeria \\ E-mail addresses: 1 Johnsono255@yahoo.com, 2 steve.adewole@uniben.edu
}

\begin{abstract}
The purpose of this study is to develop a mathematical model using Source and Green's functions for a Horizontal Wells in a Bounded Reservoir with Constant Pressure at the Top and Bottom for the interpretation of pressure responses in the reservoir based on dimensionless pressure and pressure derivative. Reservoir and well parameters investigated revealed what sets of reservoir/ well parameters combination that will prolong infinite activity of the reservoir before steady state sets in. Results show that dimensionless lateral extent does not directly affect the dimensionless pressure and dimensionless pressure derivative for very short well lengths as used in this paper. Dimensionless pressure increases with reservoir pay thickness and delay the time for steady state conditions. In fact external fluid invasion is strongly affected by the size of the pay thickness, thus the minimum time for steady state period to set in is according to the relation $T D \geq L D / 5$.
\end{abstract}

Keywords: Bounded, reservoir, steady-state conditions, horizontal well, constant pressure.

\section{INTRODUCTION}

Horizontal wells are commonly applied in oil reservoirs to enhance the production and ultimate recovery of oil and gas because of; the increased contact area of the wellbore and the pay zone and especially in constant pressure boundaries to reduce water and gas coning because of the reduced drawdown thereby reducing the remedial work required in the future [1]. However, it is very important and mandatory that pressure behavior within the well and reservoir system is properly analyzed to interpret properties associated with the system. Reservoirs with active bottom water and gas cap gas as source of energy has always been a difficult situation to handle that is why it is important that proper guidelines through appropriate mathematical models are used to modify and enhance horizontal wells to be able to achieve economic productions i.e optimally. Thus, based on the mathematical models presented parameters were investigated and their results computed in dimensionless form. Simple excel software was used to enhance computation. Determination of transient pressure behavior for horizontal wells using dimensionless approach has fully overcome the challenges faced in conventional methods and has brought about major success in pressure analysis for both conventional and horizontal wells [2]. With clear delineation of characteristic shapes from diagnostic plots, flow periods are more distinctively separated, and because most often horizontal wells are applied to reservoir to prolong infinite activities as possible dimensionless analytical approach has played a major part by simplifying and magnifying plots.

When a reservoir is bounded by water and gas cap gas, information such as pressure distribution are important in determining wellbore pressure regimes and allowable rate profiles to guarantee clean oil [3].

\section{RESERVOIR MODEL DESCRIPTION}

Figure 1 shows an isotropic rectangular boxed shaped reservoir model that is under study. It consists of a Horizontal well of dimensionless length LD in a reservoir of dimensionless thickness $\mathrm{hD}$ and is located at an elevation $z w d=0.5 \mathrm{hD}$ from the bottom boundary. 


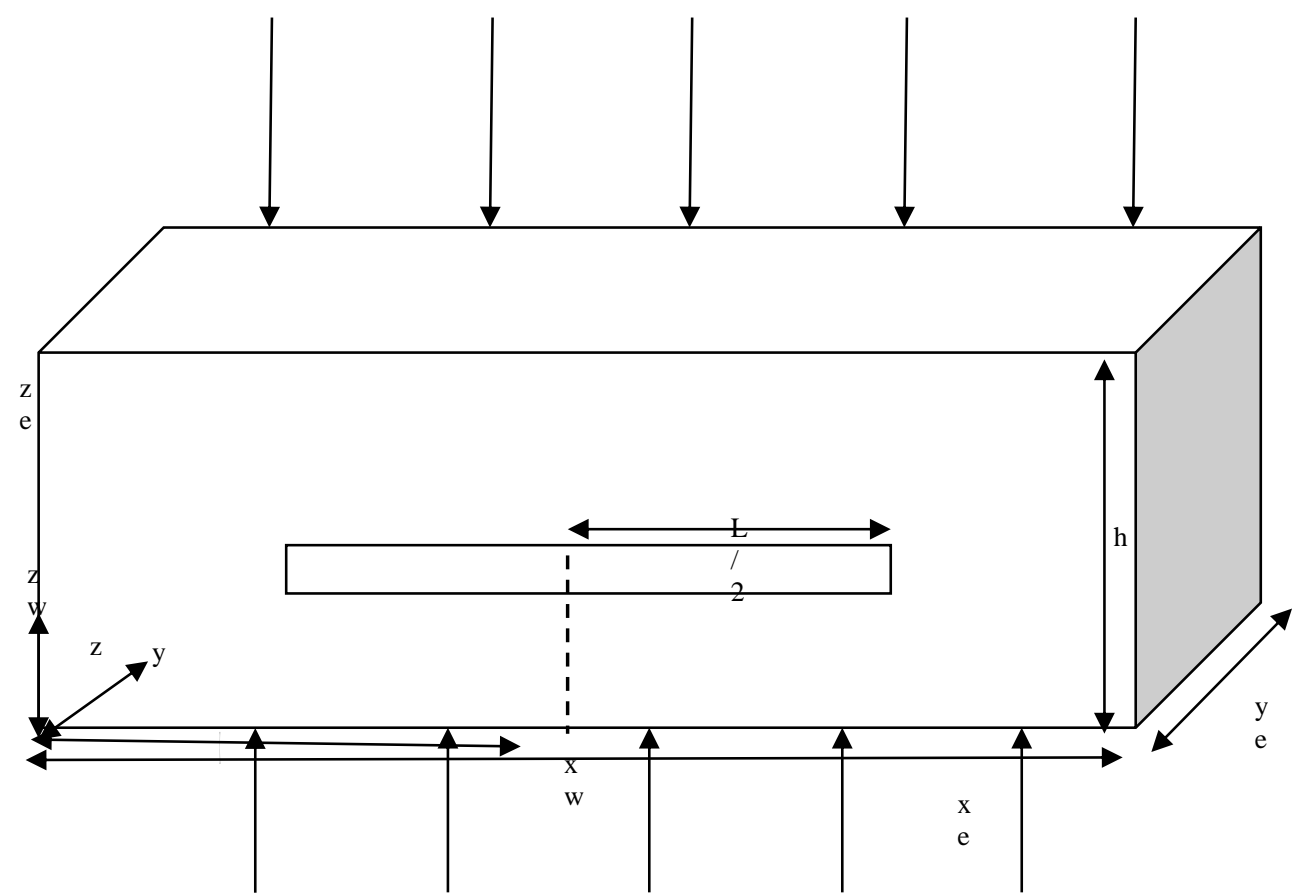

Fig. 1: Horizontal well in a bounded reservoir with constant pressure top and bottom

The reservoir is subject to constant pressure at the bottom and top (along the z-axis) boundaries, sealed laterally (along $x$ axis) and sealed along the $y$-axis. The well is assumed to be parallel to the horizontal plane of the reservoir i.e the top and bottom boundaries. Reservoir pressure is above bubble point, therefore production energy is from the expanding top gas cap gas and bottom water.

\subsection{Mathematical Model Descriptions}

The well is considered as a line source and is assumed to be producing oil at a constant rate $q$, from the reservoir at a pressure pi with a drawdown of $\Delta p$. To properly analyse this reservoir well system we assumed that; the reservoir is homogeneous, The reservoir is isotropic $\mathrm{K}_{\mathrm{x}}=\mathrm{K}_{\mathrm{y}}=\mathrm{K}_{\mathrm{z}}$, No flow boundaries, Skin, Gravitational and frictional effects are negligible. In writing down mathematical expression the concept of dimensionless analysis was employed to make computation much simply, reduce bulky expressions and provide solution to graphical representations that are simpler.

The following expression is used to compute dimensionless pressure from pressure drop:

\section{For a Horizontal Well}

$p_{D}=2 \pi h_{D} \int_{0}^{t_{D}} s\left(x_{D}, y_{D}, z_{D}, \tau\right) d \tau$

where

$$
s\left(x_{D}, y_{D}, z_{D}, t_{D}\right)=s\left(x_{D}, t_{D}\right) \cdot s\left(x_{D}, t_{D}\right) . s\left(x_{D}, t_{D}\right)(2)
$$

\subsubsection{Early time radial flow model}

During the early time radial flow period, the reservoir acts as if it is infinite acting because the transient pressure has not felt any of the reservoir boundaries The source functions expression for this flow period are as follows [4]

\section{$X$ axis}

The Source, is an infinite slab source in an infinite slab. Source function in dimensionless variables is

$$
S\left(X_{D}, t\right)=\frac{1}{2}\left[\operatorname{erf} \frac{1+X_{D}}{2 \sqrt{T_{D}}}+\operatorname{erf} \frac{1-X_{D}}{2 \sqrt{T_{D}}}\right]
$$

\section{Y axis}

Source is an infinite plane source in an infinite slab reservoir at early time

Source function

$$
S\left(Y_{D}, t\right)=\frac{1}{2 \sqrt{\pi \tau_{D}}} e^{-\frac{\left(Y_{D}-Y_{W D}\right)^{2}}{4 \tau_{D}}}
$$

\section{$Z$ axis}

Source is an infinite plane source in an infinite slab reservoir at early time

Source function number

$$
S\left(Z_{D}, t\right)=\frac{1}{2 \sqrt{\pi \tau_{D}}} e^{-\frac{\left(Z_{D}-Z_{w D}\right)^{2}}{4 \tau_{D}}}
$$

Using the Newman's product method, the dimensionless pressure response of the horizontal well during the early time radial flow period is given as 
Pressure Distribution of a Horizontal Well in a Bounded Reservoir with Constant Pressure Top and Bottom, J. J. Orene \& ES Adewole

$P_{D e}=\frac{h_{D}}{4} \int_{0}^{t_{D 1}}\left[\operatorname{erf} \frac{1+X_{D}}{2 \sqrt{\tau}}+\right.$

$\left.\left.\operatorname{erf} \frac{1-X_{D}}{2 \sqrt{\tau}}\right] \cdot \frac{1}{\tau} e^{-\frac{\left(Y_{D}-Y_{w D}\right)^{2}+\left(Z_{D}-Z_{w D}\right)^{2}}{4 \tau}}\right] d \tau$

Integrating the function in equation (6) [5]

$$
P_{D e}=\frac{-\beta}{4 L_{D}} E i\left(-\frac{\left(Z_{D}-Z_{W D}\right)^{2}}{4 t_{D}}\right)
$$

When the argument $\frac{\left(Z_{D}-Z_{W D}\right)^{2}}{4 t_{D}}$ is $\leq 0.01$ then use $X=\frac{t_{D}}{\left(Z_{D}-Z_{W D}\right)^{2}}$

Then;

$$
P_{D e}=\frac{-\beta}{4 L_{D}} \ln (1.78 x)
$$

$\beta=1$ and represent the short time approximation prior to boundary conditions

And the time limit for this flow period as given by Babu and Odeh [6] is

$$
t_{D e}=1.9008 d z^{2}
$$

Where $d z$ is the shortest distance between the well and Z-boundary

\subsubsection{Steady state flow}

This is the time where all the boundaries are felt and fluid flow from beyond the ends of the well. At steady state condition $\mathrm{dp} / \mathrm{dt}=0$ and this is the time when external fluid drive toward producing wells and the pressure at every point of the reservoir is constant.

The source functions expression for this flow period are as follows

\section{$Z$ axis}

The source is an infinite plane in an infinite slab reservoir with constant pressure boundaries at the top and bottom and the source function in dimensionless form

$$
\begin{aligned}
& S\left(Z_{D}, t_{D}\right)=\frac{2}{z_{e D}} \sum_{n=1}^{\infty} \exp \left(-\frac{n^{2} \pi^{2} t_{D}}{z_{e D}^{2}}\right) \sin \frac{n \pi z_{w}}{z_{e D}} \sin \frac{n \pi z_{D}}{z_{e D}}(10) \\
& S\left(X_{D}, t_{D}\right)=\frac{x_{f}}{x_{e D}}[1+ \\
& \left.\frac{4 x_{e D}}{\pi x_{f}} \sum_{n=1}^{\infty} \exp \left(-\frac{n^{2} \pi^{2} t_{D}}{x_{e D}^{2}}\right) \sin \frac{n \pi x_{f}}{2 x_{e D}} \cos \frac{n \pi x_{w D}}{x_{e D}} \cos \frac{n \pi x_{D}}{x_{e D}}\right](11)
\end{aligned}
$$

\section{$\mathrm{X}$ axis}

The Source, is an infinite slab source in an infinite slab. The required source

$\frac{x_{f}}{x_{e D}}[1+$

$\left.\frac{4 x_{e D}}{\pi x_{f}} \sum_{n=1}^{\infty} \exp \left(-\frac{n^{2} \pi^{2} t_{D}}{x_{e D}^{2}}\right) \sin \frac{n \pi x_{f}}{2 x_{e D}} \cos \frac{n \pi x_{w D}}{x_{e D}} \cos \frac{n \pi x_{D}}{x_{e D}}\right]$

\section{Y axis}

The Source, is an infinite plane source in an infinite slab.

The required source function
$S\left(Y_{D}, t_{D}\right)=\frac{1}{y_{e D}}[1+$

$2 \sum_{n=1}^{\infty} \exp \left(-\frac{n^{2} \pi^{2}}{y_{e D}^{2}} t_{D}\right) \cos \frac{n \pi y_{w D}}{y_{e D}} \cos \frac{n \pi y_{D}}{y_{e D}}$

Time to start this flow period according to Babu and Odeh [6]

This flow period starts at a maximum of

$t_{D l}=5.0688 \mathrm{D} x^{2} \quad$ or $t_{D l}=1.9008 \mathrm{Dz}^{2}$

Using the Newman's product method, the dimensionless pressure response of the horizontal well during the late time flow period is given as

$P_{D l}=2 \pi h \int_{t D_{e}}^{t D_{l}} \frac{1}{y_{e D}}[1+$

$2 \sum_{n=1}^{\infty} \exp \left(-\frac{n^{2} \pi^{2}}{y_{e D}^{2}} t_{D}\right) \cos \frac{n \pi y_{w D}}{y_{e D}} \cos \frac{n \pi y_{D}}{y_{e D}} *$

$\frac{2}{z_{e D}}\left[\sum_{n=1}^{\infty} \exp \left(-\frac{n^{2} \pi^{2} t_{D}}{z_{e D}^{2}}\right) \sin \frac{n \pi z_{w}}{z_{e D}} \sin \frac{n \pi z_{D}}{z_{e D}} * \frac{x_{f}}{x_{e D}}[1+\right.$

$\left.\frac{4 x_{e D}}{\pi x_{f}} \sum_{n=1}^{\infty} \exp \left(-\frac{n^{2} \pi^{2} t_{D}}{x_{e D}^{2}}\right) \sin \frac{n \pi x_{f}}{2 x_{e D}} \cos \frac{n \pi x_{w D}}{x_{e D}} \cos \frac{n \pi x_{D}}{x_{e D}}\right]$ (15)

Using Numerical integration [7] method equation (15) becomes

$P_{D l}$

$=8 h \cdot\left(\frac{t_{D l}-t_{D e}}{2}\right)\left[\sum_{1}^{N} W_{1} F\left[\frac{1}{y_{e D}}[1\right.\right.$

$+2 \sum_{n=1}^{\infty} \exp \left(-\frac{n^{2} \pi^{2}}{y_{e D}^{2}} \frac{Z_{1}\left(t_{D l}-t_{D e}\right)+\left(t_{D l}+t_{D e}\right)}{2}\right)$

$\cos \frac{n \pi x_{w D}}{y_{e D}} \cos \frac{n \pi x_{D}}{y_{e D}}$.

$\frac{2}{z_{e D}}\left[\sum_{n=1}^{\infty} \exp \left(-\frac{n^{2} \pi^{2}}{z_{e D}^{2}} \frac{Z_{1}\left(t_{D l}-t_{D e}\right)+\left(t_{D l}+t_{D e}\right)}{2}\right)\right.$

$\sin \frac{n \pi x_{w D}}{x_{e D}} \sin \frac{n \pi x_{D}}{x_{e D}} \cdot \frac{x_{f}}{x_{e D}}[1$

$+\frac{4 x_{e D}}{\pi x_{f}} \sum_{n=1}^{\infty} \exp \left(-\frac{n^{2} \pi^{2}}{x_{e D}^{2}} \cdot \frac{Z_{1}\left(t_{D l}-t_{D e}\right)+\left(t_{D l}+t_{D e}\right)}{2}\right) \sin \frac{n \pi x_{f}}{2 x_{e D}}$

$\left.\cos \frac{n \pi x_{w D}}{x_{e D}} \cos \frac{n \pi x_{D}}{x_{e D}}\right]+\sum_{1}^{N} W_{2} F\left[\frac{1}{y_{e D}}[1+\right.$

$2 \sum_{n=1}^{\infty} \exp \left(-\frac{n^{2} \pi^{2}}{y_{e D}^{2}} \frac{Z_{2}\left(t_{D l}-t_{D e}\right)+\left(t_{D l}+t_{D e}\right)}{2}\right) \cos \frac{n \pi x_{w D}}{y_{e D}} \cos \frac{n \pi x_{D}}{y_{e D}}$.

$\frac{2}{z_{e D}}\left[\sum_{n=1}^{\infty} \exp \left(-\frac{n^{2} \pi^{2}}{z_{e D}^{2}} \frac{Z_{2}\left(t_{D l}-t_{D e}\right)+\left(t_{D l}+t_{D e}\right)}{2}\right) \sin \frac{n \pi x_{w D}}{x_{e D}}\right.$ $\sin \frac{n \pi x_{D}}{x_{e D}} \cdot \frac{x_{f}}{x_{e D}}[1$

$+\frac{4 x_{e D}}{\pi x_{f}} \sum_{n=1}^{\infty} \exp \left(-\frac{n^{2} \pi^{2}}{x_{e D}^{2}} \cdot \frac{Z_{2}\left(t_{D l}-t_{D e}\right)+\left(t_{D l}+t_{D e}\right)}{2}\right) \sin \frac{n \pi x_{f}}{2 x_{e D}}$ $\left.\left.\cos \frac{n \pi x_{w D}}{x_{e D}} \cos \frac{n \pi x_{D}}{x_{e D}}\right]\right](16)$

\subsection{Dimensionless Pressure Derivatives}

The derivative approach exposes reservoir and well characteristics more specifically. The form of dimensionless pressure derivative adopted in this work is $t_{D} \frac{\partial P_{D}}{t_{D}}$. Derivations are obtained by differentiating the functions in equations (7) and (15) [8]. 
Pressure Distribution of a Horizontal Well in a Bounded Reservoir with Constant Pressure Top and Bottom, J. J. Orene \& ES Adewole During the early radial flow period, the dimensionless pressure derivative is given as;

$$
P_{D e}^{\prime}=t_{D} \frac{\partial P_{D}}{t_{D}}=\frac{-\beta}{4 L_{D}} \exp \left(\frac{\left(Z_{D}-Z_{w D}\right)^{2}}{4 \tau_{D}}\right.
$$

At late time flow period

$P_{D L}^{\prime}=t_{D} * 4 \pi * \frac{1}{y_{e D}}[1+$

$2 \sum_{n=1}^{\infty} \exp \left(-\frac{n^{2} \pi^{2}}{y_{e D}^{2}} t_{D}\right) \cos \frac{n \pi x_{w D}}{y_{e D}} \cos \frac{n \pi x_{D}}{y_{e D}} *$

$\left[\sum_{n=1}^{\infty} \exp \left(-\frac{n^{2} \pi^{2}}{z} t_{D}\right) \sin \frac{n \pi x_{w D}}{z_{e D}} \sin \frac{n \pi x_{D}}{z_{e D}} * \frac{x_{f}}{x_{e D}}[1+\right.$

$\left.\frac{4 x_{e D}}{\pi x_{f}} \sum_{n=1}^{\infty} \exp \left(-\frac{n^{2} \pi^{2} t_{D}}{x_{e D}^{2}}\right) \sin \frac{n \pi x_{f}}{2 x_{e D}} \cos \frac{n \pi x_{w D}}{x_{e D}} \cos \frac{n \pi x_{D}}{x_{e D}}\right](18)$

\section{COMPUTATION OF WELL RESPONSES:}

Dimensionless wellbore pressures and its derivative at early radial flow period are computed from Equations (6) and (16) while steady state flow period is computed by evaluating the integral numerically as presented in equation (15) and dimensionless pressure derivatives at late time is evaluated using equation (17). The following parameters were assumed to simulate wellbore environment [4]: yD = $\mathrm{ywD} ; \mathrm{m}=\mathrm{n}=1$. Furthermore, permeability $\mathrm{K}=\mathrm{Kx}=\mathrm{Ky}$ $=\mathrm{Kz}=1$.

\subsection{Effect of Reservoir Length on Dimensionless Pressure PD and dimensionless Pressure Derivatives PD'}

Assumed wellbore and reservoir parameters (set one) $\mathrm{LD}=3, \mathrm{hD}=0.33, \mathrm{ZD}=0.16998, \mathrm{ZwD}=0.1667, \mathrm{XD}=0.5$, $\mathrm{XwD}=0.25, \mathrm{YeD}=3.133, \mathrm{YD}=\mathrm{YwD}=0.3333$

Assumed wellbore and reservoir parameters (set two) $\mathrm{LD}=2, \mathrm{hD}=0.5, \mathrm{ZD}=0.253, \mathrm{ZwD}=0.25, \mathrm{XD}=0.5$, $X w D=0.25, Y e D=2.3076, Y D=Y w D=0.1538$

Different values of reservoir external lateral boundaries $\mathrm{XED}=5,10,15$ and 20 were used to

compute dimensionless pressure and dimensionless pressure derivative using equations(7), (17) at early time and equations(16) and (18) at late time and tabulated in table 1 and 2, equation 9 provide appropriate time to end the early radial flow period. Result show that different dimensionless lateral extent does not show or have significant impact on dimensionless pressure owing to the fact that other parameters are held constant thereby creating a narrow path along the reservoir in the X-direction. Usually fluid flow from the Z-direction in to wellbore after the asymptotic flow. In other word if the architecture of the reservoir is fixed and we vary parameter along the $X$, there is a minimal effect as shown in the tables below.

\subsection{The Effect of Well Length on PD and PD' for a Given Reservoir Length}

The effect of well length on dimensionless pressure was investigated, keeping XED constant at $=5$ and10, different values of $L D=0.5,1$ and 2 were investigated for their effect on dimensionless pressure and dimensionless pressure derivative using equations (7) and (17) for the infinite flow period and equations (16) and (18) for the late time flow. Result obtained shows that dimensionless well length is inversely related to dimensionless pressure and their derivatives. The higher the value of dimensionless well length the smaller the dimensionless pressure.

\section{Assumed wellbore and reservoir parameters for varying well lengths for a given XED}

$\mathrm{hD}=0.5, \mathrm{ZD}=0.255, \mathrm{ZwD}=0.25, \mathrm{XD}=0.5, \mathrm{XwD}=0.25$, $\mathrm{YeD}=4.7, \mathrm{YD}=\mathrm{YwD}=0.3333$

Table 1: Dimensionless pressure and dimensionless pressure derivatives results for external lateral extent XED investigated (set one)

\begin{tabular}{ccccccc}
\hline TD & PD,XED=5 & PD'XED=5 & PD,XED=10 & PD'XED=10 & PDXED=15 & PD'XED=15 \\
\hline 0.0001 & 0.24391667 & 0.08081993 & 0.24391667 & 0.08081993 & 0.24391667 & 0.08081993 \\
0.001 & 0.41490356 & 0.08307852 & 0.41490356 & 0.08307852 & 0.41490356 & 0.08307852 \\
0.01 & 0.60678565 & 0.08330782 & 0.60678565 & 0.08330782 & 0.60678565 & 0.08330782 \\
0.1 & 0.79866774 & 0.08333078 & 0.79866774 & 0.08333078 & 0.79866774 & 0.08333078 \\
1 & 0.79866774 & 0 & 0.79866774 & 0 & 0.79866774 & 0 \\
10 & 0.79866774 & 0 & 0.79866774 & 0 & 0.79866774 & 0 \\
100 & 0.79866774 & 0 & 0.79866774 & 0 & 0.79866774 & 0 \\
1000 & 0.79866774 & 0 & 0.79866774 & 0 & 0.79866774 & 0 \\
10000 & 0.79866774 & 0 & 0.79866774 & 0 & 0.79866774 & 0 \\
\hline
\end{tabular}


Pressure Distribution of a Horizontal Well in a Bounded Reservoir with Constant Pressure Top and Bottom, J. J. Orene \& ES Adewole

Table 2: Dimensionless pressure and dimensionless pressure derivatives results for external lateral extent XED investigated (set two)

\begin{tabular}{ccccccc}
\hline TD & PD,XED=5 & PD'XED=5 & PD,XED =10 & PD'XED =10 & PDXED=15 & PD'XED=15 \\
\hline 0.0001 & 0.40225 & 0.1222189 & 0.40225 & 0.1222189 & 0.40225 & 0.1222189 \\
0.001 & 0.66089301 & 0.12471907 & 0.66089301 & 0.12471907 & 0.66089301 & 0.12471907 \\
0.01 & 0.94871614 & 0.12497188 & 0.94871614 & 0.12497188 & 0.94871614 & 0.12497188 \\
0.1 & 1.23653928 & 0.12499719 & 1.23653928 & 0.12499719 & 1.23653928 & 0.12499719 \\
1 & 1.560134 & $2.7088 \mathrm{E}-17$ & 1.710489 & $1.568 \mathrm{E}-17$ & 2.075653 & $1.4063 \mathrm{E}-17$ \\
10 & 1.560134 & $1.419 \mathrm{E}-169$ & 1.710489 & $1.023 \mathrm{E}-169$ & 2.075653 & $1.053 \mathrm{E}-169$ \\
100 & 1.560134 & 0 & 1.710489 & 0 & 2.075653 & 0 \\
1000 & 1.560134 & 0 & 1.710489 & 0 & 2.075653 & 0 \\
10000 & 1.560134 & 0 & 1.710489 & 0 & 2.075653 & 0 \\
\hline
\end{tabular}

Table 3 Dimensionless pressure and dimensionless pressure derivatives results for varying well lengths $L D$ when $X E D=5$

\begin{tabular}{ccccccc}
\hline TD & $P D, L D=2$ & $P D ' L D=2$ & $P D, L D=1$ & $P D ' L D=1$ & $P D, L D=0.5$ & $P^{\prime} D^{\prime} L D=0.5$ \\
\hline 0.0001 & 0.281125 & 0.1174266 & 0.56225 & 0.2348533 & 1.1245 & 0.4697065 \\
0.001 & 0.5331866 & 0.1242212 & 1.0663732 & 0.2484424 & 2.132746409 & 0.4968847 \\
0.01 & 0.8210097 & 0.1249219 & 1.6420195 & 0.2498438 & 3.284038956 & 0.4996876 \\
0.1 & 1.1088329 & 0.1249922 & 2.2176658 & 0.2499844 & 4.435331502 & 0.4999688 \\
1 & 1.2499 & $1.76 \mathrm{E}-18$ & 2.4998 & $9.72 \mathrm{E}-06$ & 4.999601 & 0.014902 \\
10 & 1.2499 & $4.6 \mathrm{E}-171$ & 2.4998 & $1.25 \mathrm{E}-43$ & 4.999601 & $8.7 \mathrm{E}-12$ \\
100 & 1.2499 & 0 & 2.4998 & 0 & 4.999601 & $2.2 \mathrm{E}-106$ \\
1000 & 1.2499 & 0 & 2.4998 & 0 & 4.999601 & 0 \\
10000 & 1.2499 & 0 & 2.4998 & 0 & 4.999601 & 0 \\
\hline
\end{tabular}

Table 4: Dimensionless pressure and dimensionless pressure derivatives results for varying well lengths $L D$ when XED $=10$

\begin{tabular}{ccccccc}
\hline TD & PD, LD=2 & PD'LD=2 & $P D, L D=1$ & $P D ' L D=1$ & $P D, L D=0.5$ & $P^{\prime} L D=0.5$ \\
\hline 0.0001 & 0.281125 & 0.117427 & 0.56225 & 0.234854 & 1.124500 & 0.49875156 \\
0.001 & 0.533187 & 0.124221 & 1.0663732 & 0.2484424 & 2.132746 & 0.49987502 \\
0.01 & 0.821009 & 0.124920 & 1.64201948 & 0.2498438 & 3.284039 & 0.4999875 \\
0.1 & 1.108833 & 0.124992 & 2.21766575 & 0.2499844 & 4.43533 & 0.49999875 \\
1 & 1.295379 & $1.28 \mathrm{E}-17$ & 2.590758 & $1.193 \mathrm{E}-05$ & 5.181517 & 0.00245445 \\
10 & 1.295379 & $4.6 \mathrm{E}-170$ & 2.590758 & $7.70 \mathrm{E}-171$ & 5.181517 & $1.4735 \mathrm{E} 171$ \\
100 & 1.295379 & 0 & 2.590758 & 0 & 5.181517 & 0 \\
1000 & 1.295379 & 0 & 2.590758 & 0 & 5.181517 & 0 \\
10000 & 1.295379 & 0 & 2.590758 & 0 & 5.181517 & 0 \\
\hline
\end{tabular}

\subsection{The Effect of Dimensionless Reservoir Height on PD and PD'}

Keeping other assumed parameters constant as shown in table above different values of dimensionless pay thickness $\mathrm{hD}=0.5,1$ and 2 was investigated at XED $=5$ and 10 and results tabulated in Tables 3 and 4. It was observed that dimensionless pay thickness has effect on dimensionless pressure and dimensionless pressure derivative. The higher the values of $\mathrm{hD}$ the larger the PD and hence the better the productivity of the well/reservoir.

\section{DISCUSSION OF RESULTS}

Dimensionless pressure and dimensionless pressure derivatives were computed for the various reservoir and well parameters. At early radial flow period equations (7) and (17) was utilized and equations (16) and (18) was used for the computation at late time, and the results presented in tables. Result from tables presented above show that there is a progressive rise in $P D$ at early time till at $T D \geq L D / 5$, this is a characteristic signature of an infinite acting flow period. Dimensionless Pressure derivative results further confirm this behaviour with constant value of 
Pressure Distribution of a Horizontal Well in a Bounded Reservoir with Constant Pressure Top and Bottom, J. J. Orene \& ES Adewole

$1 / 4$ LD. Beyond this time the reservoir is said to have started producing away from the tip of the well and this time period is referred to as the late time flow period or as the time period when steady state is setting in. Results from Pressure derivatives shows as the values gradually collapse to zero. This is a typical example of a reservoir with constant pressure boundaries. Although the time to reach steady state flow period varies for parameters but $h D \geq 1$ will generally delay steady state condition.

Results from tables 3 and 4 also shows that dimensionless well length is inversely related to dimensionless pressure and dimensionless pressure derivative. This is an indication that longer well length will produce more crude in less time whereas short well lengths will prolong production. The implication is that using short well length will prolong production but there is a very big risk of external fluid infiltration and possible fingering earlier than expected.

\section{CONCLUSION}

The effect of reservoir and well parameters on dimensionless pressure and dimensionless pressure derivatives distribution of a horizontal well in a bounded reservoir with constant pressure top and bottom have been studied. The following conclusions can be made;

- Shorter dimensionless well lengths have more effect on dimensionless pressure and pressure derivatives i. e. efficiency in production and thus optimal production is achieved with shorter well lengths at early time, but at late time as $\mathrm{dp} / \mathrm{dt}$ approaches zero so does external fluid encroachment toward wellbore

- Larger Dimensionless pay thickness are more productive than shorter ones

- Dimensionless external lateral extent also increases productivity of the system. But for very short well lengths $L D<<20$ and thus pay thickness the effect of XEDs are insignificant at late time because of the presence of constant pressure boundaries

- Well length and pay thickness are the two parameters that determine time to reach steady state.

\section{DIFINITION OF PARAMETERS}

$$
\begin{aligned}
& i_{D}=\frac{2 i}{l} \\
& L_{D}=\frac{L}{2 h}
\end{aligned}
$$

$$
\begin{aligned}
& r_{w D}=z_{D}-z_{w D} \\
& t_{D}=\frac{0.001056 k t}{\emptyset \mu c_{t} l^{2}} \\
& t_{D}=\frac{k h \Delta p}{141.2 q u b}
\end{aligned}
$$

\section{NOMENCLATURES}

$\mathrm{hD}=$ Dimensionless reservoir height or pay thickness $\mathrm{kx}=$ permeability in the $\mathrm{x}$ direction in the areal plane, $\mathrm{md}$

ky = permeability in the $y$ direction in the areal plane, $\mathrm{md}$

$\mathrm{kv}=\mathrm{kz}=$ permeability in the $\mathrm{z}$ direction. It is the permeability in the direction

perpendicular to the bedding which controls flow due to gravity, md

$\mathrm{k}$; = Permeability in the i direction

$\mathrm{L}=$ horizontal well length, $\mathrm{ft}$

$\mathrm{LD}=$ dimensionless well length

$M=n$ number of image wells

$\mathrm{PD}=$ dimensionless pressure

$\mathrm{P}^{\prime} \mathrm{De}=$ dimensionless pressure derivative at early time

$\mathrm{P}^{\prime} \mathrm{DI}=$ dimensionless pressure derivative at late time

$r w D=$ dimensionless wellbore radius

$\mathrm{S}=$ skin factor

$\mathrm{t}=$ flow time, hrs

$\mathrm{tD}=$ dimensionless time

$\mathrm{tD}=$ Dimensionless time

tDe $=$ Dimensionless time at early time flow

$\mathrm{tDI}=$ Dimensionless time at late time flow

W $=$ weight associated with Gaussian Quadrature

$x e=$ reservoir length

$x e D=$ dimensionless reservoir length

$\mathrm{XD}=$ perforation length

ye $=$ reservoir width

yeD $=$ dimensionless reservoir width

ze $=$ Vertical distance between drainhole no-flow boundaries

$\mathrm{zW}=$ vertical distance from the wellbore to the bottom boundary (well stand-off)

$\mathrm{zWD}=$ Dimensionless vertical distance from the wellbore to the bottom boundary (well stand-off)

Z=root associated with Gaussian Quadrature

\section{REFERENCES}

[1]. S. Al Rbeawi and D. Tiab; 'Transient Pressure Analysis of Horizontal Wells in a Multi-Boundary System',American Journal of Engineering Research, Vol. 2, Issue 04, 2013, pp 44-66.

[2]. I. Eiroboyi, and S. I. Wilkie.; 'Comparative Evaluation of Pressure Distribution between 
Pressure Distribution of a Horizontal Well in a Bounded Reservoir with Constant Pressure Top and Bottom, J. J. Orene \& ES Adewole

Horizontal and Vertical Wells in a Reservoir (Edge Water Drive)' , Nigerian Journal of Technology (NIJOTECH) Vol. 36, No. 2, April 2017, pp. $457-460$

[3]. Matthews, C. S. and Russell, D.G."Pressure Buildup and Flow test in Wells. Monograh Series", Society of Petroleum Engineers of AIME, Dallas, 1967.

[4]. A.C. Gringarten. The Use of Source and Green's Functions in Solving Unsteady -

State Flow Problems in Reservoirs'. Society of Petroleum Engineers Journal, pages 285\{296, Oct. 1973. pp 285-295.

[5]. Adewole, E. S and Bello, K. O. "Application Gauss- Laguerre Quadrature in Computing
Exponential Integral Function, (Ei)."Paper SPE 98829 presented at the Society of Petroleum Engineers Annual Conference and Exhibition held in Abuja, Nigeria (2005) 1-7.

[6]. Babu, D.K and Odeh, A.S. 'Transient Flow Behavior of Horizontal Wells; Pressure drawdown and Buildup Analysis," SPE Formation Evaluation (March 1990), pp 7-15.

[7]. Carnahan, B. Luther, H.A. and Wilkes, J.O. "Applied Numerical Methods", John Wiley and Sons, 1969, p. 69- 140.

[8]. Stroud, K. A: "Engineering Mathematics (Fifth Edition)". Industrial Press, Inc. New York. 